\title{
Generic Properties of Stochastic Entropy Production
}

\author{
Simone Pigolotti ${ }^{1,2},{ }^{*}$ Izaak Neri ${ }^{1,3},^{\dagger}$ Édgar Roldán $^{1},^{\ddagger}$ and Frank Jülicher ${ }^{1,4 \S}$ \\ 1 Max Planck Institute for the Physics of Complex Systems, Nöthnitzerstraße 38, 01187 Dresden, Germany \\ 2 Biological Complexity Unit, Okinawa Institute for Science and \\ Technology and Graduate University, Onna, Okinawa 904-0495, Japan \\ ${ }^{3}$ Max Planck Institute of Molecular Cell Biology and Genetics, \\ Pfotenhauerstraße 108, 01307 Dresden, Germany \\ ${ }^{4}$ Center for Systems Biology Dresden, Pfotenhauerstraße 108, 01307 Dresden, Germany
}

\begin{abstract}
We derive an Itô stochastic differential equation for entropy production in nonequilibrium Langevin processes. Introducing a random-time transformation, entropy production obeys a onedimensional drift-diffusion equation, independent of the underlying physical model. This transformation allows us to identify generic properties of entropy production. It also leads to an exact uncertainty equality relating the Fano factor of entropy production and the Fano factor of the random time, which we also generalize to non steady-state conditions.

PACS numbers: 05.70.Ln, 05.40.-a, 02.50.Le
\end{abstract}

The laws of thermodynamics can be extended to mesoscopic systems [1-5]. For such systems, energy changes on the order of the thermal energy $k_{\mathrm{B}} T$ are relevant. Here, $k_{\mathrm{B}}$ is the Boltzmann constant and $T$ the temperature. Therefore, thermodynamic observables associated with mesoscopic degrees of freedom are stochastic. A key example of such thermodynamics observables is the stochastic entropy production in nonequilibrium processes. Recent experimental advances in micromanipulation techniques permit the measurement of stochastic entropy production in the laboratory [6-10].

Certain statistical properties of stochastic entropy production are generic, i.e., they are independent of the physical details of a system. Examples of such generic properties are the celebrated fluctuation theorems, for reviews see $[2,4,5]$. Recently, it was shown that infima and passage probabilities of entropy production are also generic [11]. Other statistical properties of entropy production are system-dependent, such as the mean value [12-15], the variance [16, 17], the first-passage times of entropy production [18-20] and the large deviation function [21, 22]. Nevertheless, these properties are sometimes constrained by universal bounds $[11,14,16$, 17, 23-27]. It remains unclear which statistical properties of stochastic entropy production are generic, and why.

In this Letter, we introduce a theoretical framework which addresses this question for nonequilibrium Langevin processes. We identify generic properties of entropy production by their independence of a stochastic variable $\tau$ which we call entropic time. We find that the evolution of steady-state entropy production as a function of $\tau$ is governed by a simple one-dimensional driftdiffusion process, independent of the underlying model. This allows us to identify a set of generic properties of entropy production and obtain exact results characterizing entropy production fluctuations.

We consider a mesoscopic system described by $n$ slow degrees of freedom $\vec{X}=\left(X_{1}(t), X_{2}(t), \ldots, X_{n}(t)\right)^{\top}$. The system is in contact with a thermostat at temperature $T$. The stochastic dynamics of the system can be described by the probability distribution $P(\vec{X}, t)$ to find the system in a configuration $\vec{X}$ at time $t$. This probability distribution satisfies the Smoluchowski equation

$$
\partial_{t} P=-\vec{\nabla} \cdot \vec{J}
$$

where the probability current is given by

$$
\vec{J}=\boldsymbol{\mu} \cdot \vec{F} P-\boldsymbol{D} \cdot \vec{\nabla} P
$$

Here we have introduced the force at time $t, \vec{F}=$ $-\vec{\nabla} U(\vec{X}(t), t)+\vec{f}(\vec{X}(t), t)$, where $U$ is a potential and $\vec{f}$ is a non-conservative force. We always imply no flux or periodic boundary conditions. The state-dependent mobility and diffusion tensors, $\boldsymbol{\mu}(\vec{X}(t))$ and $\boldsymbol{D}(\vec{X}(t))$ respectively, are symmetric and obey the Einstein relation $\boldsymbol{D}=k_{\mathrm{B}} T \boldsymbol{\mu}$. This system can also be represented by a Langevin equation with multiplicative noise as [21, 28]

$$
\frac{\mathrm{d} \vec{X}}{\mathrm{~d} t}=\boldsymbol{\mu} \cdot \vec{F}+\vec{\nabla} \cdot \boldsymbol{D}+\sqrt{2} \boldsymbol{\sigma} \cdot \vec{\xi}
$$

Here $\vec{\xi}(t)=\left(\xi_{1}(t), \xi_{2}(t), \ldots, \xi_{n}(t)\right)^{\top}$ is a Gaussian white noise with mean $\left\langle\xi_{i}(t)\right\rangle=0$ and autocorrelation $\left\langle\xi_{i}(t) \xi_{j}\left(t^{\prime}\right)\right\rangle=\delta_{i j} \delta\left(t-t^{\prime}\right)$ where $\langle\ldots\rangle$ denotes an ensemble average. Here and throughout the paper the noise terms are interpreted in the Itô sense. The tensor $\boldsymbol{\sigma}$ obeys $\boldsymbol{\sigma} \boldsymbol{\sigma}^{\top}=\boldsymbol{D}$ and can be chosen as $\boldsymbol{\sigma}=\boldsymbol{D}^{1 / 2}$. In the Itô interpretation, the term $\vec{\nabla} \cdot \boldsymbol{D}$ is required for consistency with Eqs. (1) and (2) as it compensates a noise-induced drift [29]. Examples of systems described by Eq. (31) that we consider in this paper are represented in Fig. 1: a colloidal particle driven by a constant force along a one-dimensional periodic potential (Fig. 1A); a colloidal particle in a two-dimensional non-conservative force field pointing in the $x$ direction (Fig. 1B); and a chiral active Brownian motion in two dimensions [30] (Fig. 1C). 

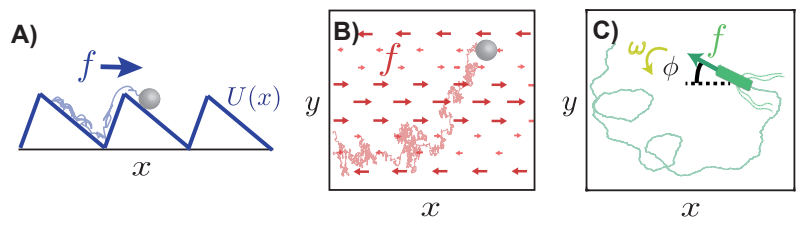

FIG. 1. Examples of nonequilibrium steady states. (A) Brownian particle driven by a constant non-conservative force in a periodic $1 \mathrm{D}$ sawtooth potential, $\mathrm{d} X / \mathrm{d} t=\mu\left[f-\partial_{X} U(X)\right]+$ $\sqrt{2 D} \xi$, with the potential $U(x)=\left(U_{0} x\right) / x^{*}$ for $x \in\left[0, x^{*}\right]$ and $U(x)=U_{0}(1-x) /\left(1-x^{*}\right)$ for $x \in\left[x^{*}, 1\right]$. (B) $2 \mathrm{D}$ transport in a force field: $\mathrm{d} X / \mathrm{d} t=\mu f \cos (2 \pi Y)+\sqrt{2 D} \xi_{x}$ and $\mathrm{d} Y / \mathrm{d} t=\sqrt{2 D} \xi_{y}$. (C) Chiral active Brownian motion described by 3 degrees of freedom: position coordinates $\mathrm{d} X / \mathrm{d} t=\mu f \cos (\phi)+\sqrt{2 D} \xi_{x}, \mathrm{~d} Y / \mathrm{d} t=\mu f \sin (\phi)+\sqrt{2 D} \xi_{y}$ and orientation angle $\mathrm{d} \phi / \mathrm{d} t=\mu_{\phi} \omega+\sqrt{2 D_{\omega}} \xi_{\omega}$. In (B) and (C) $U=0$ and $f$ is an external non-conservative force.

We now discuss the stochastic thermodynamics of the process described by Eq. (3). In Itô's calculus, the rate of change of the potential $U(\vec{X}(t), t)$ is given by Itô's lemma [31]:

$$
\frac{\mathrm{d} U}{\mathrm{~d} t}=\partial_{t} U+\vec{\nabla} U(\vec{X}(t), t) \cdot \frac{\mathrm{d} \vec{X}}{\mathrm{~d} t}+\operatorname{Tr}[\boldsymbol{D} \cdot \vec{\nabla} \vec{\nabla} U]
$$

where $\operatorname{Tr}$ denotes the trace and the dots denote tensor contractions. In stochastic thermodynamics, the first law can be expressed as $\mathrm{d} U=\mathrm{d} W+\mathrm{d} Q$, where $\mathrm{d} W$ is the work performed on the system and $\mathrm{d} Q$ is the mesoscopic heat exchanged with the thermostat during a time interval $\mathrm{d} t$ [1]. In Itô's calculus, the rates of change of work and heat are given by [21]

$$
\begin{aligned}
& \frac{\mathrm{d} W}{\mathrm{~d} t}=\partial_{t} U+\vec{f} \cdot \frac{\mathrm{d} \vec{X}}{\mathrm{~d} t}+\operatorname{Tr}[\boldsymbol{D} \cdot \vec{\nabla} \vec{f}] \\
& \frac{\mathrm{d} Q}{\mathrm{~d} t}=-\vec{F} \cdot \frac{\mathrm{d} \vec{X}}{\mathrm{~d} t}-\operatorname{Tr}[\boldsymbol{D} \cdot \vec{\nabla} \vec{F}]
\end{aligned}
$$

The expressions (5) and (6) are the Itô versions of the stochastic work and mesoscopic heat originally defined by Sekimoto using the Stratonovich interpretation $[1,32]$.

We define the stochastic entropy production $S_{\text {tot }} / k_{\mathrm{B}}$ as the logarithm of the ratio of probabilities of forward and time-reversed stochastic trajectories $[3,21,33]$. This definition is equivalent to $\mathrm{d} S_{\text {tot }} / \mathrm{d} t=-(1 / T) \mathrm{d} Q / \mathrm{d} t-$ $k_{\mathrm{B}} \mathrm{d} \ln P(\vec{X}(t), t) / \mathrm{d} t$, where the first term can be interpreted as an exchange of entropy with the reservoir and the second term as a change of system entropy. Using Eq. (6) and Itô's lemma, as in Eq. (4) (see [34]), we obtain the following Itô stochastic differential equation for the entropy production rate

$$
\frac{\mathrm{d} S_{\mathrm{tot}}}{\mathrm{d} t}=-2 k_{\mathrm{B}} \partial_{t} \ln P+v_{\mathrm{S}}+\sqrt{2 k_{\mathrm{B}} v_{\mathrm{S}}} \xi_{S}
$$

Here we define the entropic drift $v_{S}(\vec{X}(t), t) \geq 0$ as

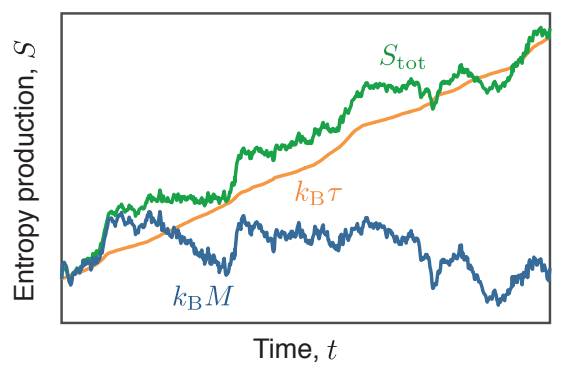

FIG. 2. Illustration of the decomposition of stochastic entropy production. In nonequilibrium steady states, the stochastic entropy production $S_{\text {tot }}(t) / k_{\mathrm{B}}$ (green) is given by the sum of the monotonously increasing entropic time $\tau(t)$ (orange), and the martingale process $M(t)$ (blue), see Eq. (11).

$$
v_{S}=k_{\mathrm{B}} \frac{\vec{J} \cdot \boldsymbol{D}^{-1} \cdot \vec{J}}{P^{2}},
$$

which on average equals the average rate of entropy production, $\left\langle v_{S}\right\rangle=\left\langle\mathrm{d} S_{\text {tot }} / \mathrm{d} t\right\rangle[5,28]$. Entropy fluctuations are governed by the noise term $\xi_{S}=\vec{\xi} \cdot \sigma^{-1}$. $\vec{J} / \sqrt{\vec{J} \cdot \boldsymbol{D}^{-1} \cdot \vec{J}}$ which is a one-dimensional Gaussian white noise with $\left\langle\xi_{S}(t)\right\rangle=0$ and $\left\langle\xi_{S}(t) \xi_{S}\left(t^{\prime}\right)\right\rangle=\delta\left(t-t^{\prime}\right)$. The Itô Eq. (7) is equivalent to the Langevin equation for entropy production in the Stratonovich interpretation given in Ref. [33]. For each trajectory generated by Eq. (3), Eq. (7) generates the corresponding entropy production. From Eq. (7) we can derive several generic properties of stochastic entropy production in nonequilibrium processes.

We first discuss properties of nonequilibrium steady states for which $\partial_{t} P=0$. We now calculate the time derivative of $e^{-S_{\mathrm{tot}} / k_{\mathrm{B}}}$ in steady state. Using Itô's lemma, we obtain from Eq. (7)

$$
\frac{\mathrm{d} e^{-S_{\mathrm{tot}} / k_{\mathrm{B}}}}{\mathrm{d} t}=-\sqrt{2 \frac{v_{S}}{k_{\mathrm{B}}}} e^{-S_{\mathrm{tot}} / k_{\mathrm{B}}} \xi_{S}
$$

which reveals that $e^{-S_{\text {tot }} / k_{\mathrm{B}}}$ is a geometric Brownian motion with zero drift and time-dependent diffusion coefficient. The fact that $e^{-S_{\text {tot }} / k_{\mathrm{B}}}$ has no drift implies that $e^{-S_{\mathrm{tot}} / k_{\mathrm{B}}}$ is a martingale process $[11,31,38]$. Using $S_{\mathrm{tot}}(0)=0$ the integral fluctuation theorem $\left\langle e^{-S_{\text {tot }}(t) / k_{\mathrm{B}}}\right\rangle=1$ follows immediately from Eq. (9).

In steady state, Eq. (7) can be simplified by introducing the dimensionless entropic time

$$
\tau=\frac{1}{k_{\mathrm{B}}} \int_{0}^{t} v_{S}\left(\vec{X}\left(t^{\prime}\right)\right) \mathrm{d} t^{\prime}
$$

which is an example of a random time [31]. Note that, in steady state, $v_{S}(\vec{X}(t), t)=v_{S}(\vec{X}(t))$ represents the expected rate of entropy production at a given point in phase space $\vec{X}(t)$ and $\tau$ thus represents the accumulated expected entropy production. In nonequilibrium situations with $v_{S}>0$, the entropic time $\tau(t)$ is monotonously 

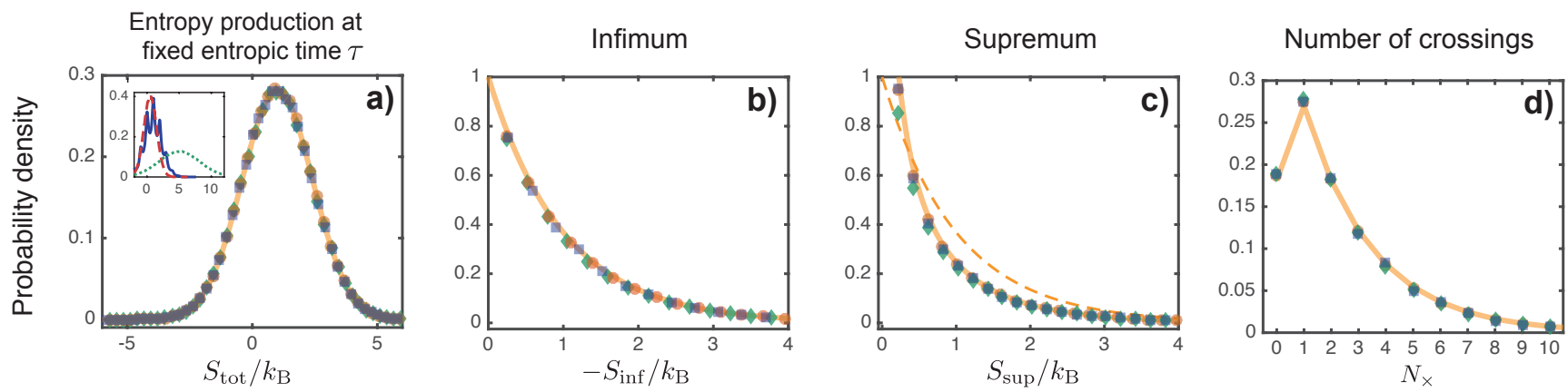

FIG. 3. Generic properties of stochastic entropy production. Distributions of a) entropy production at fixed $\tau=1$, (b) infimum of entropy production, c) supremum of entropy production before the infimum, d) number of crossings of entropy production, with $\Delta=0.2 k_{\mathrm{B}}$. The symbols are obtained from numerical simulations of the three models sketched in Fig. 1 (blue squares, model A; red circles, model B; green diamonds, model C). The inset of a) shows numerically estimated distributions of $S_{\text {tot }}$ for the three models at fixed $t=1$ for comparison. The solid orange curves are the theoretical expressions a) a Gaussian distribution with average $k_{\mathrm{B}} \tau$ and variance $2 k_{\mathrm{B}}^{2} \tau$ b) an exponential distribution with average $-k_{\mathrm{B}}$ c) Eq. (13); d) Eq. (14). The dashed line in c) is the theoretical distribution of minus the infimum for comparison. In all simulations, parameters are $\boldsymbol{\mu}=\mathbb{I}$, $\boldsymbol{D}=k_{\mathrm{B}} T \mathbb{I}$, where $\mathbb{I}$ is the identity matrix, and $f=1$. In Model A we chose $U_{0}=k_{\mathrm{B}} T$ and $x^{*}=0.3$. In model $\mathrm{C}$ we chose $\omega=2$. Here and in the following figures, each point represents an average over $10^{6}$ simulations.

increasing with $t$. Integrating Eq. (7) we obtain

$$
S_{\mathrm{tot}}(t) / k_{\mathrm{B}}=\tau(t)+M(t) .
$$

Equation (11) represents the decomposition of entropy production into a monotonously increasing process $\tau(t)$ and a martingale $M(t)=\sqrt{2 / k_{\mathrm{B}}} \int_{0}^{t} \sqrt{v_{S}\left(\vec{X}\left(t^{\prime}\right)\right)} \xi_{S}\left(t^{\prime}\right) \mathrm{d} t^{\prime}$ that has zero mean, $\langle M(t)\rangle=0$, as illustrated in Fig. 2 . This decomposition is unique and is known as the DoobMeyer decomposition [39].

We now discuss an important implication of Eqs. (7) and (10). Performing the random-time transformation $t \rightarrow \tau$ in Eq. (7) we obtain a Langevin equation for steady state entropy production at entropic times [31]

$$
\frac{1}{k_{\mathrm{B}}} \frac{\mathrm{d} S_{\mathrm{tot}}}{\mathrm{d} \tau}=1+\sqrt{2} \eta(\tau)
$$

where $\eta(\tau(t))=\sqrt{k_{\mathrm{B}} / v_{S}(\vec{X}(t))} \xi_{S}(t)$ such that $\eta(\tau)$ is Gaussian white noise with $\langle\eta(\tau)\rangle=0$ and $\left\langle\eta(\tau) \eta\left(\tau^{\prime}\right)\right\rangle=$ $\delta\left(\tau-\tau^{\prime}\right)$. Equation (12) states that a temporal trajectory of entropy production of any nonequilibrium steady state can be mapped to a trajectory of a drift-diffusion process with constant drift $k_{\mathrm{B}}$ and diffusion coefficient $k_{\mathrm{B}}^{2}$, where the mapping consists in a time-dependent, stochastic contraction or dilation of time. This implies that all properties of $S_{\text {tot }}$ that are invariant under such transformation are generic.

One such property is the distribution of entropy production at fixed values of $\tau$, which must be a Gaussian with average $k_{\mathrm{B}} \tau$ and variance $2 k_{\mathrm{B}}^{2} \tau$ because of Eq. (12). This is indeed the case for all three model examples, see Fig. 3a. Note that the distribution of entropy production at fixed time $t$ are very different for the three models, as shown in the inset of Fig. 3a. Another generic property is the distribution of the global infimum of entropy production $S_{\text {inf }}$, previously derived using martingale theory [11] and given by an exponential distribution $P\left(S_{\mathrm{inf}}\right)=e^{S_{\mathrm{inf}} / k_{\mathrm{B}}} / k_{\mathrm{B}}$ with mean $-k_{\mathrm{B}}$ and $S_{\mathrm{inf}} \leq 0$ (Fig. $3 \mathrm{~b}$ ). Also the supremum of entropy production before the infimum is generic and distributed according to

$$
P\left(S_{\mathrm{sup}}\right)=2 e^{S_{\mathrm{sup}} / k_{\mathrm{B}}} \operatorname{acoth}\left(2 e^{S_{\mathrm{sup}} / k_{\mathrm{B}}}-1\right)-1
$$

with $S_{\text {sup }} \geq 0$. Its average value is $\left\langle S_{\text {sup }}\right\rangle=\left(\pi^{2} / 6-\right.$ 1) $k_{\mathrm{B}} \approx 0.645 k_{\mathrm{B}}$. The number of times that entropy production crosses a given threshold value is also generic. An example is the number of times $N_{\times}$that entropy production crosses from $-\Delta$ to $\Delta$ with $\Delta>0$. The distribution of $N_{\times}$is

$$
P\left(N_{\times} ; \Delta\right)= \begin{cases}1-e^{-\Delta / k_{\mathrm{B}}} & N_{\times}=0 \\ 2 \sinh \left(\Delta / k_{\mathrm{B}}\right) e^{-2 N_{\times} \Delta / k_{\mathrm{B}}} & N_{\times} \geq 1\end{cases}
$$

Equation (13) and (14) are in excellent agreement with numerical simulations, as shown in Fig. 3c,d.

With equation (11), we can also compute the moments of $S_{\text {tot }}(t)$. The first moment reads simply $\left\langle S_{\text {tot }}\right\rangle=k_{\mathrm{B}}\langle\tau\rangle$. The second moment is $\left\langle S_{\text {tot }}^{2}\right\rangle=2 k_{\mathrm{B}}^{2}\langle\tau\rangle+k_{\mathrm{B}}^{2}\left\langle\tau^{2}\right\rangle$, see [34]. Combining these two results, the Fano factor of the entropy production can be expressed as

$$
\frac{1}{k_{\mathrm{B}}} \frac{\sigma_{S_{\mathrm{tot}}}^{2}}{\left\langle S_{\mathrm{tot}}\right\rangle}=2+\frac{\sigma_{\tau}^{2}}{\langle\tau\rangle},
$$

where $\sigma_{y}^{2}=\left\langle y^{2}\right\rangle-\langle y\rangle^{2}$ denotes the variance. The thermodynamic Fano factor equality given by Eq. (15) is an exact relation, valid for finite times, between the fluctuations of entropy production and the fluctuations of the entropic time $\tau$. This equation provides further physical 


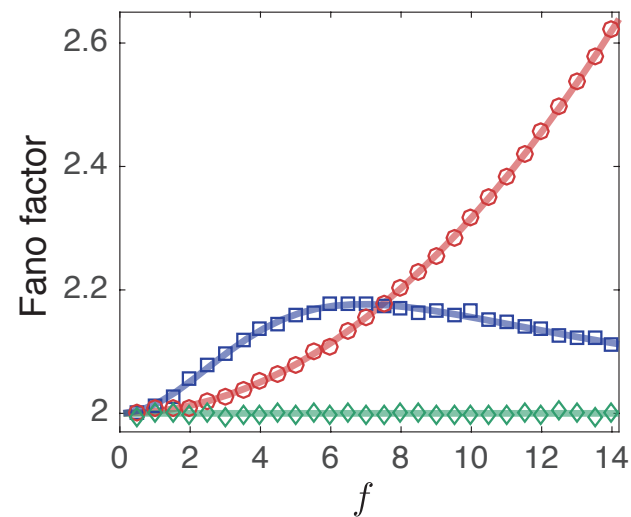

FIG. 4. Thermodynamic Fano factor equality. Long-time Fano factor of entropy production $\sigma_{S_{\text {tot }}}^{2} /\left(k_{\mathrm{B}}\left\langle S_{\mathrm{tot}}\right\rangle\right)$ as a function of the external force $f$. The symbols are obtained from numerical simulations of the models shown in Fig. 1A (blue), Fig. 1B (red), and Fig. 1C (green). The solid lines are the prediction of Eq. (15) and have been calculated by means of Eq. (16) (see [34]). All the parameters of the numerical simulations except of the external force $f$ are the same as in Fig. 3.

insight into the previously introduced finite-time uncertainty relation, $\sigma_{S_{\text {tot }}}^{2} /\left\langle S_{\text {tot }}\right\rangle \geq 2 k_{\mathrm{B}}[26,27]$. The variance obeys the equality $\sigma_{S_{\text {tot }}}^{2} /\left\langle S_{\text {tot }}\right\rangle=2 k_{\mathrm{B}}$ only if the entropic time satisfies $\sigma_{\tau}^{2} /\langle\tau\rangle \stackrel{=}{=}$, which holds e.g. near equilibrium. In this case, the distribution of entropy production is Gaussian. Another example for which $\sigma_{\tau}^{2} /\langle\tau\rangle=0$ is the chiral active Brownian motion shown in Fig. 1c.

For long times, the variance of the entropic time can be estimated by a Green-Kubo formula as an integral over a correlation function [34]

$$
\frac{\sigma_{\tau}^{2}}{\langle\tau\rangle}=\frac{2}{k_{\mathrm{B}}\left\langle v_{S}\right\rangle} \int_{0}^{\infty} \mathrm{d} t^{\prime}\left(\left\langle v_{S}\left(\vec{X}\left(t^{\prime}\right)\right) v_{S}(\vec{X}(0))\right\rangle-\left\langle v_{S}\right\rangle^{2}\right) .
$$

Using Equations (15) and (16) we obtain explicit expressions for the Fano factor as a function of the driving force for our three models, see Fig. 4 for a comparison with numerical simulations.

Our theory can also be applied to nonequilibrium processes out of steady state. From Eq. (7) we derive the general Fano factor equality

$$
\frac{1}{k_{\mathrm{B}}} \frac{\sigma_{S_{\mathrm{tot}}}^{2}}{\left\langle S_{\mathrm{tot}}\right\rangle}=2+\frac{\sigma_{\tau}^{2}}{\langle\tau\rangle}+\frac{2 \Omega}{\langle\tau\rangle}
$$

where

$\Omega=\frac{1}{k_{\mathrm{B}}} \int_{0}^{t} \mathrm{~d} t^{\prime} \int_{0}^{t^{\prime}} \mathrm{d} t^{\prime \prime}\left\langle-2 \partial_{t^{\prime \prime}} \ln P\left(X\left(t^{\prime \prime}\right), t^{\prime \prime}\right) v_{S}\left(\vec{X}\left(t^{\prime}\right), t^{\prime}\right)\right\rangle$

and $\tau=\left(1 / k_{\mathrm{B}}\right) \int_{0}^{t} v_{S}\left(\vec{X}\left(t^{\prime}\right), t^{\prime}\right) \mathrm{d} t^{\prime}$ is the entropic time for non-steady state processes. At steady state, $\Omega=0$, and Eq. (17) reduces to Eq. (15). Note that the argument of the integral in Eq. (18) is the correlation of the two drift
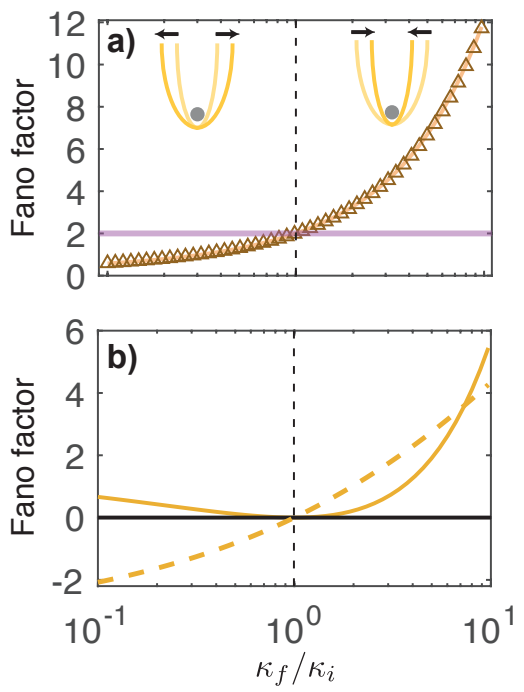

FIG. 5. Fano factor of stochastic entropy production out of steady state. The position of a Brownian particle is governed by the equation $\mathrm{d} X / \mathrm{d} t=-\mu \kappa_{f} X+\sqrt{2 D} \xi$ with $\mu=1$ and $D=\mu k_{\mathrm{B}} T$. The particle is initially at equilibrium with stiffness $\kappa_{i}=1$ (see inset). a) Comparison between the exact value (orange line) of the long-time Fano factor of entropy production and the value obtained from numerical simulations (brown triangles) as a function of $\kappa_{f}$. The exact value is given by $\sigma_{S_{\text {tot }}}^{2} / k_{\mathrm{B}}\left\langle S_{\text {tot }}\right\rangle=\left(\kappa_{f} / \kappa_{i}-1\right)^{2} /\left[\left(\kappa_{f} / \kappa_{i}-1\right)-\log \left(\kappa_{f} / \kappa_{i}\right)\right]$ see Supplemental Material for details [34]. In simulations we measure $\langle\tau\rangle, \sigma_{\tau}^{2}$ and $\Omega$ and use Eq. (17). The horizontal purple line is set to 2 for comparison. b) Behaviour of $\sigma_{\tau}^{2} /\langle\tau\rangle$ (solid line) and $2 \Omega /\langle\tau\rangle$ (dashed line) as a function of $\kappa_{f}$.

terms in Eq. (7) at different times. In Fig. 5, we illustrate Eq. (17) for a particle confined in a harmonic trap, where the stiffness of the trap is instantaneously quenched from a value $\kappa_{i}$ to a value $\kappa_{f}$. When $\kappa_{f}>\kappa_{i}$, one has $\Omega>0$, so that the Fano factor of entropy production is larger than two according to Eq. (17). When instead $\kappa_{f}<\kappa_{i}$, one has $\Omega<0$, and the Fano factor of entropy production is lower than two.

For nonequilibrium processes starting at thermal equilibrium and undergoing a defined protocol to a final state, one has $T S_{\text {tot }}=W-\Delta F$, where $W$ is the work performed during the protocol and $\Delta F$ is the change of equilibrium free energy $F=\langle U\rangle_{\text {eq }}+k_{\mathrm{B}} T\langle\ln P\rangle_{\text {eq }}$ associated with the final and initial states $[40-42]$. Here $\langle\cdot\rangle_{\text {eq }}$ denotes an equilibrium average over the Boltzmann distribution. For such protocols, Eq. (17) implies

$$
\Delta F=\langle W\rangle-\frac{\sigma_{W}^{2}}{2 k_{\mathrm{B}} T}+\frac{k_{\mathrm{B}} T}{2}\left(\sigma_{\tau}^{2}+2 \Omega\right) .
$$

Note that $\Delta F$ also obeys Jarzynski's equality $\Delta F=$ $-k_{\mathrm{B}} T \ln \left\langle e^{-W / k_{\mathrm{B}} T}\right\rangle[40]$, which has the form of a cumulant generating function. Comparing it with Eq. (19), one can relate the term in parenthesis in (19) to a sum of cumulants of $W / k_{\mathrm{B}} T$ of order three and higher. This sum vanishes if the work distribution is Gaussian [40]. 
We have shown that, in steady-state Langevin processes, entropy production is governed by a Langevin equation which only depends on the system's details via the entropic drift $v_{S}$. As a consequence all systemspecific features of stochastic entropy production can be absorbed into a single stochastic quantity, the entropic time $\tau$. Entropy productions of different systems at equal entropic time have the same statistics, and all properties independent of the entropic time are generic. Fluctuations of the entropic time uniquely determine the Fano factor of entropy production, providing physical insight for previously obtained bounds [16, 17, 23-27].

We have demonstrated our results for coupled overdamped Langevin equations but expect our results to hold more generally for continuous processes, as is the case for the infimum of entropy production [11]. Using the Doob-Meyer decomposition of entropy production, our definition of entropic time can also be generalized to underdamped systems [43, 44] and jump processes [45]. Our results can be experimentally tested for example with optical tweezers [6-8, 46, 47], feedback traps [9], single-electron transistors [10] and light-activated phototactic microparticles [48].

We thank AC Barato for stimulating discussions and A Mazzino and A Vulpiani for suggesting the example of model B.

* simone.pigolotti@oist.jp

† izaakneri@posteo.net

‡ edgar@pks.mpg.de

$\S$ julicher@pks.mpg.de

[1] K. Sekimoto, Stochastic energetics, vol. 799 (Springer, 2010).

[2] C. Bustamante, J. Liphardt, and F. Ritort, Physics Today 58, 43 (2005).

[3] C. Maes, F. Redig, and A. V. Moffaert, J. Math. Phys. 41, 1258 (2000).

[4] C. Jarzynski, Eur. Phys. J. B 64, 331 (2008).

[5] U. Seifert, Rep. Prog. Phys. 75, 126001 (2012).

[6] I. A. Martínez, É. Roldán, L. Dinis, and R. A. Rica, Soft Matter 13, 22 (2017).

[7] J. R. Gomez-Solano, A. Petrosyan, S. Ciliberto, and C. Maes, J. Stat. Mech. 2011, P01008 (2011).

[8] T. Speck, V. Blickle, C. Bechinger, and U. Seifert, EPL 79, 30002 (2007).

[9] M. Gavrilov, R. Chétrite, and J. Bechhoefer, arXiv:1703.07601 (2017).

[10] J. V. Koski, A. Kutvonen, I. M. Khaymovich, T. AlaNissila, and J. P. Pekola, Phys. Rev. Lett. 115, 260602 (2015).

[11] I. Neri, E. Roldán, and F. Jülicher, Phys. Rev. X 7, 011019 (2017).

[12] S. Bo, and A. Celani, Phys. Rep. 670, 1 (2016).

[13] T. Harada, and S.-i. Sasa, Phys. Rev. Lett. 95, 130602 (2005).

[14] R. Kawai, J. M. R. Parrondo, and C. Van den Broeck, Phys. Rev. Lett. 98, 080602 (2007).
[15] J. M. R. Parrondo, C. Van den Broeck, and R. Kawai, New J. Phys. 11, 073008 (2009).

[16] A. C. Barato and U. Seifert, Phys. Rev. Lett. 114, 158101 (2015).

[17] P. Pietzonka, A. C. Barato, and U. Seifert, Phys. Rev. E 93, 052145 (2016).

[18] É. Roldán, I. Neri, M. Dörpinghaus, H. Meyr, and F. Jülicher, Phys. Rev. Lett. 115, 250602 (2015).

[19] K. Saito and A. Dhar, EPL 114, 50004 (2016).

[20] T. R. Gingrich, and J. M. Horowitz, arXiv:1706.09027 (2017).

[21] J. L. Lebowitz and H. Spohn, J. Stat. Phys. 95, 333 (1999).

[22] J. Mehl, T. Speck, and U. Seifert, Phys. Rev. E 78, 011123 (2008).

[23] T. R. Gingrich, J. M. Horowitz, N. Perunov, and J. L. England, Phys. Rev. Lett. 116, 120601 (2016).

[24] M. Polettini, A. Lazarescu, and M. Esposito, Phys. Rev. E 94, 052104 (2016).

[25] J. P. Garrahan, Phys. Rev. E 95, 032134 (2017).

[26] P. Pietzonka, F. Ritort, and U. Seifert, Phys. Rev. E 96, 012101 (2017).

[27] T. R. Gingrich, and J. M. Horowitz, arXiv:1707.03805 (2017).

[28] C. Maes, K. Netočnỳ, and B. Wynants, Phys. A 387, 2675 (2008).

[29] A. W. Lau and T. C. Lubensky, Phys. Rev. E 76, 011123 (2007).

[30] C. Bechinger, R. Di Leonardo, H. Löwen, C. Reichhardt, G. Volpe, and G. Volpe, Rev. Mod. Phys. 88, 045006 (2016).

[31] B. Øksendal, Stochastic differential equations: an introduction with applications (Springer Science \& Business Media, 2013).

[32] K. Sekimoto, Prog. Theor. Phys. Suppl. 130, 17 (1998).

[33] U. Seifert, Phys. Rev. Lett. 95, 040602 (2005).

[34] See Supplemental Material, which includes Refs. [35-37], for details on mathematical derivations of the Langevin equation for entropy production, the Fano factor equalities and the explicit calculation of the Fano factor for the three models.

[35] J. L. Doob, Bull. Soc. Math. Fra. 85, 431 (1957).

[36] S. N. Majumdar and H. Orland, J. Stat. Mech. 6, 06039 (2015).

[37] R. Chetrite and H. Touchette, Ann. Math. Poincare 16, 2005 (2015).

[38] R. Chetrite and S. Gupta, J. Stat. Phys. 143, 543 (2011).

[39] R. Liptser and A. N. Shiryaev, Statistics of random Processes: I. general Theory, vol. 5 (Springer, 2013).

[40] C. Jarzynski, Phys. Rev. Lett. 78, 2690 (1997).

[41] G. E. Crooks, Phys. Rev. E 60, 2721 (1999).

[42] G. Bochkov and Y. E. Kuzovlev, Zh. Eksp. Teor. Fiz 72, 238 (1977).

[43] A. Celani, S. Bo, R. Eichhorn, and E. Aurell, Phys. Rev. Lett. 109, 260603 (2012).

[44] H. Ge, Phys. Rev. E 89, 022127 (2014).

[45] P. Gaspard, J. Stat. Phys. 117, 599 (2004).

[46] S. Krishnamurthy, S. Ghosh, D. Chatterji, R. Ganapathy, and A. K. Sood, Nature Phys. 12, 1134 (2016).

[47] A. Argun, A. Moradi, E. Pinçe, G. Baris Bagci, A. Imparato and G. Volpe, Phys. Rev. E 94, 062150 (2016).

[48] C. Lozano, B. ten Hagen, H. Löwen, and C. Bechinger, Nat. Commun. 7, 12828 (2016). 


\section{SUPPLEMENTAL MATERIAL}

This document provides additional information for the manuscript "Generic Properties of Stochastic Entropy Production". It is organized as follows. Section S1 sketches the derivation of the Itô stochastic differential equation for the entropy production. Section S2 presents a derivation of the Fano-factor equality for steady-state processes. Sections S3A, S3B, and S3C detail the calculations of the Fano factor of entropy production for the three steady-state models discussed in the Main Text. Section S4 presents the derivation of the Fano-factor equality out of steady state. Section S5 describes details on the non-equilibrium process shown in Fig. 5 of the Main text.

\section{S1. ITO STOCHASTIC DIFFERENTIAL EQUATION FOR ENTROPY PRODUCTION}

In this section, we sketch the derivation of the evolution for the stochastic entropy production (Eq. (7) in the Main Text). We recall that the rate of total entropy production change can be decomposed into the rates of system-entropy change and heat change [33]

$$
\frac{\mathrm{d} S_{\mathrm{tot}}}{\mathrm{d} t}=\frac{\mathrm{d} S_{\mathrm{sys}}}{\mathrm{d} t}-\frac{1}{T} \frac{\mathrm{d} Q}{\mathrm{~d} t}
$$

with $S_{\mathrm{sys}}=-k_{B} \ln P$ the system entropy. We express the rate of heat change as

$$
\begin{aligned}
-\frac{1}{k_{\mathrm{B}} T} \frac{\mathrm{d} Q}{\mathrm{~d} t} & =\frac{\vec{F}}{k_{\mathrm{B}} T} \cdot \frac{\mathrm{d} \vec{X}}{\mathrm{~d} t}+\frac{1}{k_{\mathrm{B}} T} \operatorname{Tr}[\boldsymbol{D} \cdot \vec{\nabla} \vec{F}] \\
& =\frac{1}{k_{\mathrm{B}} T} \vec{F} \cdot(\boldsymbol{\mu} \cdot \vec{F}+\vec{\nabla} \cdot \boldsymbol{D})+\operatorname{Tr}[\boldsymbol{\mu} \cdot \vec{\nabla} \vec{F}]+\frac{1}{k_{\mathrm{B}} T} \sqrt{2}(\vec{F} \cdot \boldsymbol{\sigma} \cdot \vec{\xi}),
\end{aligned}
$$

where we have used the Einstein relation $\boldsymbol{D}=k_{\mathrm{B}} T \boldsymbol{\mu}$. We use Itô's lemma [31] to find the following expressions for the rate of system-entropy change

$$
\begin{aligned}
& k_{\mathrm{B}}^{-1} \frac{\mathrm{d} S_{\mathrm{sys}}}{\mathrm{d} t}=-\frac{\mathrm{d}}{\mathrm{d} t} \ln P \\
& =-\frac{\partial_{t} P}{P}-\frac{\vec{\nabla} P}{P} \cdot \frac{\mathrm{d} \vec{X}}{\mathrm{~d} t}+\operatorname{Tr}\left[\boldsymbol{D} \cdot\left[\frac{\vec{\nabla} P \vec{\nabla} P}{(P)^{2}}-\frac{\vec{\nabla} \vec{\nabla} P}{P}\right]\right] \\
& =-\frac{\partial_{t} P}{P}-\frac{\vec{\nabla} P}{P} \cdot(\boldsymbol{\mu} \cdot \vec{F}+\vec{\nabla} \cdot \boldsymbol{D})+\operatorname{Tr}\left[\boldsymbol{D} \cdot \frac{\vec{\nabla} P \vec{\nabla} P}{(P)^{2}}\right]-\operatorname{Tr}\left[\boldsymbol{D} \cdot \frac{\vec{\nabla} \vec{\nabla} P}{P}\right]-\sqrt{2} \operatorname{Tr}\left[\boldsymbol{\sigma}^{T} \cdot \frac{\vec{\nabla} P}{P} \vec{\xi}\right] .
\end{aligned}
$$

The Fokker-Planck equation can be rewritten as

$$
\begin{aligned}
& \operatorname{Tr}\left[\boldsymbol{D} \cdot \frac{\vec{\nabla} \vec{\nabla} P}{P}\right]+(\vec{\nabla} \cdot \boldsymbol{D}) \cdot \frac{\vec{\nabla} P}{P} \\
& =\frac{\partial_{t} P}{P}+\operatorname{Tr}\left[\boldsymbol{\mu} \cdot\left(\vec{F} \frac{\vec{\nabla} P}{P}\right)\right]+\operatorname{Tr}[\boldsymbol{\mu} \cdot \vec{\nabla} \vec{F}]+\frac{1}{T} \vec{F} \cdot(\vec{\nabla} \cdot \boldsymbol{D}) .
\end{aligned}
$$

After substituting equation (23) into the expression for the rate of system-entropy change, Eq. (22), and adding the rate of heat change, Eq. (21), we obtain the following compact expression for the rate of entropy production change

$$
\frac{\mathrm{d} S_{\mathrm{tot}}}{\mathrm{d} t}=-2 k_{\mathrm{B}} \partial_{t} \ln P+v_{\mathrm{S}}+\sqrt{2 k_{\mathrm{B}} v_{\mathrm{S}}} \xi_{S}
$$

with $v_{S}=k_{\mathrm{B}} \vec{J} \cdot \boldsymbol{D}^{-1} \cdot \vec{J} / P^{2}, \xi_{S}=\vec{\xi} \cdot \boldsymbol{\sigma}^{-1} \cdot \vec{J} / \sqrt{\vec{J} \cdot \boldsymbol{D}^{-1} \cdot \vec{J}}$, and the probability currents $\vec{J}=\boldsymbol{\mu} \cdot \vec{F} P-\boldsymbol{D} \cdot \vec{\nabla} P$.

\section{S2. FANO FACTOR OF ENTROPY PRODUCTION}

In this section we derive the Fano-factor equality for stochastic entropy production $S_{\text {tot }}$ at finite times

$$
\frac{\sigma_{S_{\mathrm{tot}}}^{2}}{k_{\mathrm{B}}\left\langle S_{\mathrm{tot}}\right\rangle}=2+\frac{\sigma_{\tau}^{2}}{\langle\tau\rangle}
$$


We show that (25) holds for steady state processes $\vec{X}(t)$ satisfying the Langevin Eq. (3) in the Main Text. The Langevin equation for entropy production (24) implies that the steady-state stochastic entropy production $S_{\text {tot }}(t)$ can be expressed as

$$
\frac{S_{\mathrm{tot}}(t)}{k_{\mathrm{B}}}=\tau(t)+M(t)
$$

where $\tau(t)=k_{\mathrm{B}}^{-1} \int_{0}^{t} v_{S}\left(\vec{X}\left(t^{\prime}\right)\right) \mathrm{d} t^{\prime}, M(t)=\sqrt{2 / k_{\mathrm{B}}} \int_{0}^{t} \mathrm{~d} t^{\prime} \sqrt{v_{S}\left(\vec{X}\left(t^{\prime}\right)\right)} \xi_{S}\left(t^{\prime}\right)$ and we recall that $v_{S}(\vec{X})=k_{\mathrm{B}} \vec{J} \cdot \boldsymbol{D}^{-1} \cdot \vec{J} / P^{2}$. Taking the average of Eq. (26), we find

$$
\frac{\left\langle S_{\mathrm{tot}}(t)\right\rangle}{k_{\mathrm{B}}}=\langle\tau(t)\rangle=\frac{t\left\langle v_{S}(\vec{X}(t))\right\rangle}{k_{\mathrm{B}}}
$$

From Eq. (26) we find for the second moment of stochastic entropy production

$$
\begin{aligned}
\frac{\left\langle S_{\text {tot }}^{2}(t)\right\rangle}{k_{\mathrm{B}}^{2}} & =\left\langle\left[\frac{1}{k_{\mathrm{B}}} \int_{0}^{t} \mathrm{~d} t^{\prime} v_{S}\left(\vec{X}\left(t^{\prime}\right)\right)+\sqrt{\frac{2}{k_{\mathrm{B}}}} \int_{0}^{t} \mathrm{~d} t^{\prime} \xi_{S}\left(t^{\prime}\right) \sqrt{v_{S}\left(\vec{X}\left(t^{\prime}\right)\right)}\right]^{2}\right\rangle \\
& =2\langle\tau(t)\rangle+\left\langle\tau^{2}(t)\right\rangle+2 \sqrt{\frac{2}{k_{\mathrm{B}}^{3}}}\left\langle\int_{0}^{t} \mathrm{~d} t^{\prime} \int_{0}^{t} \mathrm{~d} t^{\prime \prime} \xi_{S}\left(t^{\prime \prime}\right) v_{S}\left(\vec{X}\left(t^{\prime}\right)\right) \sqrt{v_{S}\left(\vec{X}\left(t^{\prime \prime}\right)\right)}\right\rangle
\end{aligned}
$$

where the contribution $2\langle\tau(t)\rangle$ has been obtained using Ito's isometry [31]. Subtracting $\langle\tau\rangle^{2}$ from both sides, further dividing by $\langle\tau\rangle$ and using Eq. (27) we obtain

$$
\frac{\sigma_{S_{\mathrm{tot}}}^{2}}{k_{\mathrm{B}}\left\langle S_{\mathrm{tot}}\right\rangle}=2+\frac{\sigma_{\tau}^{2}}{\langle\tau\rangle}+\frac{2}{\langle\tau\rangle} \sqrt{\frac{2}{k_{\mathrm{B}}^{3}}}\left\langle\int_{0}^{t} \mathrm{~d} t^{\prime} \int_{0}^{t} \mathrm{~d} t^{\prime \prime} \xi_{S}\left(t^{\prime \prime}\right) v_{S}\left(\vec{X}\left(t^{\prime}\right)\right) \sqrt{v_{S}\left(\vec{X}\left(t^{\prime \prime}\right)\right)}\right\rangle
$$

We show below that

$$
I\left(t^{\prime \prime}, t^{\prime}\right)=\left\langle\xi_{S}\left(t^{\prime \prime}\right) v_{S}\left(\vec{X}\left(t^{\prime}\right)\right) \sqrt{v_{S}\left(\vec{X}\left(t^{\prime \prime}\right)\right)}\right\rangle=0
$$

for stationary processes. Therefore the Fano-factor equality (15) for stochastic entropy production follows from Eq. (29). To show (30) we first note that for $t^{\prime \prime} \geq t^{\prime}$ the relation (30) is a direct consequence of the rules of Itô calculus. This is because the noise in the future is uncorrelated with the trajectory in the past. The case of $t^{\prime \prime}<t^{\prime}$ requires a careful analysis, since we have to average over the noise $\xi_{S}\left(t^{\prime \prime}\right)$ conditioned on $v_{S}\left(\vec{X}\left(t^{\prime}\right)\right)$ at a future time.

To compute this conditioned average, we apply Doob's h-transform [35] (see also [36, 37]). In short, Doob's htransform maps a stochastic process with noise variables conditioned on a future event to a stochastic process with unconditioned noise variables, but with an additional drift term. For example, consider the Langevin equation

$$
\frac{\mathrm{d} \vec{X}}{\mathrm{~d} t}=\boldsymbol{\mu} \cdot \vec{F}+\vec{\nabla} \cdot \boldsymbol{D}+\sqrt{2} \boldsymbol{\sigma} \cdot \vec{\xi}
$$

with $\langle\vec{\xi}(t)\rangle=0$ and $\left\langle\xi_{i}(t) \xi_{j}\left(t^{\prime}\right)\right\rangle=\delta_{i j} \delta\left(t-t^{\prime}\right)$. We calculate averages conditioned on the future constraint $\vec{X}\left(t^{*}\right)=x^{*}$, with $t^{*}>t$. In other words, when taking averages we only consider the trajectories generated by (31) for which $\vec{X}\left(t^{*}\right)=\vec{x}^{*}$, and disregard the other ones. In general, averages involving the noise variables $\xi_{i}(t)$ become biased by this condition, i.e., $\left.\langle\vec{\xi}(t)\rangle\right|_{\vec{X}\left(t^{*}\right)=\vec{x}^{*}} \neq 0$. Introducing the $h$-function, $h\left(\vec{x}, t ; \vec{x}^{*}, t^{*}\right)=P\left(\vec{x}^{*}, t^{*} \mid \vec{x}(t), t\right)$, where $P\left(\vec{x}, t \mid \vec{x}_{0}, t_{0}\right)$ is the solution of the corresponding Fokker-Planck equation with initial condition $\vec{x}\left(t_{0}\right)=\vec{x}_{0}$. Doob's h-transform generates a Langevin equation for a process $\vec{Z}(t)$ which reads [35-37]

$$
\frac{\mathrm{d} \vec{Z}}{\mathrm{~d} t}=\boldsymbol{\mu} \cdot \vec{F}+\vec{\nabla} \cdot \boldsymbol{D}+2 \boldsymbol{D} \cdot \vec{\nabla} \ln h\left(\vec{Z}, t ; \vec{x}^{*}, t^{*}\right)+\sqrt{2} \boldsymbol{\sigma} \cdot \vec{\eta}
$$

where $\vec{\nabla} \ln h\left(\vec{x}_{1}, t_{1} ; \vec{x}_{2}, t_{2}\right)=\vec{\nabla}_{\vec{x}_{1}} \ln h\left(\vec{x}_{1}, t_{1} ; \vec{x}_{2}, t_{2}\right)$ and $\vec{\eta}(t)$ is a white noise with zero mean, i.e., $\langle\vec{\eta}(t)\rangle=0$. Doob showed that (32) generates an ensemble of trajectories $\{\vec{Z}(t)\}_{t \in\left[0, t^{*}\right]}$ identical to the ensemble of trajectories $\{\vec{X}(t)\}_{t \in\left[0, t^{*}\right]}$ generated by the stochastic differential equation (31) and conditioned on the event $\vec{X}\left(t^{*}\right)=\vec{x}^{*}$ 
in the future [35-37]. Comparing Eq. (31) with Eq. (32), reveals that replacing the noise in Eq. (31) with the noise process defined by

$$
\vec{\xi}(t)=\vec{\eta}(t)+\sqrt{2} \sigma^{\top} \cdot \vec{\nabla} \ln h\left(\vec{Z}(t), t ; \vec{x}^{*}, t^{*}\right)
$$

allows to use standard noise averages when calculating averages conditioned on the future event at time $t^{\star}$. Note that in (33) we have used $\boldsymbol{\sigma} \cdot \boldsymbol{\sigma}^{\top}=\boldsymbol{D}$.

Using the Doob $h$-transform, we compute now the average of Eq. (30) for $t^{\prime}>t^{\prime \prime}$

$$
\begin{aligned}
I\left(t^{\prime \prime}, t^{\prime}\right) & =\left\langle\xi_{S}\left(t^{\prime \prime}\right) \sqrt{v_{S}\left(\vec{X}\left(t^{\prime \prime}\right)\right)} v_{S}\left(\vec{X}\left(t^{\prime}\right)\right)\right\rangle \\
& =\left\langle\frac{\vec{\xi}\left(t^{\prime \prime}\right) \cdot \boldsymbol{\sigma}^{-1} \cdot \vec{J}\left(t^{\prime \prime}\right)}{\sqrt{\vec{J}\left(t^{\prime \prime}\right) \cdot \boldsymbol{D}^{-1} \cdot \vec{J}\left(t^{\prime \prime}\right)}} \sqrt{v_{S}\left(\vec{X}\left(t^{\prime \prime}\right)\right)} v_{S}\left(\vec{X}\left(t^{\prime}\right)\right)\right\rangle \\
& =\sqrt{2}\left\langle\frac{\left[\vec{\nabla} \ln h\left(\vec{Z}\left(t^{\prime \prime}\right), t^{\prime \prime} ; \vec{X}\left(t^{\prime}\right), t^{\prime}\right)\right] \cdot \vec{J}\left(t^{\prime \prime}\right)}{\sqrt{\vec{J}\left(t^{\prime \prime}\right) \cdot \boldsymbol{D}^{-1} \cdot \vec{J}\left(t^{\prime \prime}\right)}} \sqrt{v_{S}\left(\vec{Z}\left(t^{\prime \prime}\right)\right)} v_{S}\left(\vec{X}\left(t^{\prime}\right)\right)\right\rangle
\end{aligned}
$$

where $h\left(\vec{Z}\left(t^{\prime \prime}\right), t^{\prime \prime} ; \vec{X}\left(t^{\prime}\right), t^{\prime}\right)=P\left(\vec{Z}\left(t^{\prime}\right), t^{\prime} \mid \vec{Z}\left(t^{\prime \prime}\right), t^{\prime \prime}\right)$ is the $h$-function for the future condition $Z\left(t^{\prime}\right)=X\left(t^{\prime}\right)$. Note that we have used $\left\langle f\left[\vec{Z}\left(t^{\prime}\right), \vec{Z}\left(t^{\prime \prime}\right)\right] \vec{\eta}\left(t^{\prime \prime}\right)\right\rangle=0$, which follows from the fact that $\vec{Z}\left(t^{\prime}\right)$ is not fluctuating and $\vec{\eta}$ is a white noise which is uncorrelated with $\vec{Z}$ at the same time. We also used $\boldsymbol{\sigma} \cdot \boldsymbol{\sigma}^{-1}=\mathbf{1}$. We proceed by writing the average $\langle\ldots\rangle$ explicitly in terms of the distribution of the system states $\vec{x}^{\prime}$ and $\vec{x}^{\prime \prime}$ at times $t^{\prime}$ and $t^{\prime \prime}$ :

$$
\begin{aligned}
I\left(t^{\prime \prime}, t^{\prime}\right)= & \sqrt{2 k_{\mathrm{B}}} \int \mathrm{d} \vec{x}^{\prime} \int \mathrm{d} \vec{x}^{\prime \prime} P\left(\vec{x}^{\prime \prime}, t^{\prime \prime}\right) P\left(\vec{x}^{\prime}, t^{\prime} \mid \vec{x}^{\prime \prime}, t^{\prime \prime}\right) \\
& \times \frac{\left[\vec{\nabla}_{\vec{x}^{\prime \prime}} \ln h\left(\vec{x}^{\prime \prime}, t^{\prime \prime} ; \vec{x}^{\prime}, t^{\prime}\right)\right] \cdot \vec{J}\left(t^{\prime \prime}\right)}{\sqrt{\vec{J}\left(t^{\prime \prime}\right) \cdot \boldsymbol{D}^{-1} \cdot \vec{J}\left(t^{\prime \prime}\right)}} \frac{\sqrt{\vec{J}\left(t^{\prime \prime}\right) \cdot \boldsymbol{D}^{-1} \cdot \vec{J}\left(t^{\prime \prime}\right)}}{P\left(\vec{x}^{\prime \prime}, t^{\prime \prime}\right)} v_{S}\left(\vec{x}^{\prime}, t^{\prime}\right) \\
= & \sqrt{2 k_{\mathrm{B}}} \int \mathrm{d} \vec{x}^{\prime} \int \mathrm{d} \vec{x}^{\prime \prime} P\left(\vec{x}^{\prime}, t^{\prime} \mid \vec{x}^{\prime \prime}, t^{\prime \prime}\right)\left[\vec{\nabla}_{\vec{x}^{\prime \prime}} \ln h\left(\vec{x}^{\prime \prime}, t^{\prime \prime} ; \vec{x}^{\prime}, t^{\prime}\right)\right] \cdot \vec{J}\left(t^{\prime \prime}\right) v_{S}\left(\vec{x}^{\prime}, t^{\prime}\right) .
\end{aligned}
$$

Using $h\left(\vec{x}^{\prime \prime}, t^{\prime \prime} ; \vec{x}^{\prime}, t^{\prime}\right)=P\left(\vec{x}^{\prime}, t^{\prime} \mid \vec{x}^{\prime \prime}, t^{\prime \prime}\right)$, we integrate by parts:

$$
\begin{aligned}
I\left(t^{\prime \prime}, t^{\prime}\right) & =\sqrt{2 k_{\mathrm{B}}} \int \mathrm{d} \vec{x}^{\prime} \int \mathrm{d} \vec{x}^{\prime \prime}\left[\vec{\nabla}_{\vec{x}^{\prime \prime}} P\left(\vec{x}^{\prime}, t^{\prime} \mid \vec{x}^{\prime \prime}, t^{\prime \prime}\right)\right] \cdot \vec{J}\left(t^{\prime \prime}\right) v_{S}\left(\vec{x}^{\prime}, t^{\prime}\right) \\
& =-\sqrt{2 k_{\mathrm{B}}} \int \mathrm{d} \vec{x}^{\prime} \int \mathrm{d} \vec{x}^{\prime \prime} P\left(\vec{x}^{\prime}, t^{\prime} \mid \vec{x}^{\prime \prime}, t^{\prime \prime}\right)\left(\vec{\nabla}_{\vec{x}^{\prime \prime}} \cdot \vec{J}\left(t^{\prime \prime}\right)\right) v_{S}\left(\vec{x}^{\prime}, t^{\prime}\right) \\
& =\sqrt{2 k_{\mathrm{B}}} \int \mathrm{d} \vec{x}^{\prime} \int \mathrm{d} \vec{x}^{\prime \prime} P\left(\vec{x}^{\prime}, t^{\prime} \mid \vec{x}^{\prime \prime}, t^{\prime \prime}\right) \partial_{t^{\prime \prime}} P\left(\vec{x}^{\prime \prime}, t^{\prime \prime}\right) v_{S}\left(\vec{x}^{\prime}, t^{\prime}\right) \\
& =0
\end{aligned}
$$

In the second step, we have used that no boundary term arise for periodic or no flux boundary conditions. Indeed either the flux or the difference in probability must vanish for this boundary conditions. In steady state $I\left(t^{\prime}, t^{\prime \prime}\right)=0$ because $\partial_{t^{\prime \prime}} P\left(\vec{x}^{\prime \prime}, t^{\prime \prime}\right)=0$.

\section{S3. FANO FACTOR OF THE ENTROPIC TIME}

The Fano factor of the entropic time $\tau$ at finite times can be expressed as

$$
\frac{\sigma_{\tau}^{2}}{\langle\tau\rangle}=\frac{\int_{0}^{t} \mathrm{~d} t^{\prime} \int_{-t^{\prime}}^{t-t^{\prime}} \mathrm{d} t^{\prime \prime}\left\langle v_{S}\left(\vec{X}\left(t^{\prime}\right)\right) v_{S}\left(\vec{X}\left(t^{\prime}+t^{\prime \prime}\right)\right)\right\rangle-\left\langle v_{S}\left(\vec{X}\left(t^{\prime}\right)\right)\right\rangle^{2}}{k_{\mathrm{B}} t\left\langle v_{S}\right\rangle},
$$

that in the limit $t \rightarrow \infty$ reduces to the Green-Kubo-like expression

$$
\frac{\sigma_{\tau}^{2}}{\langle\tau\rangle}=\frac{2}{k_{\mathrm{B}}\left\langle v_{S}\right\rangle} \int_{0}^{\infty} \mathrm{d} t^{\prime \prime}\left\langle v_{S}\left(\vec{X}\left(t^{\prime}+t^{\prime \prime}\right)\right) v_{S}\left(\vec{X}\left(t^{\prime}\right)\right)\right\rangle-\left\langle v_{S}\left(\vec{X}\left(t^{\prime}\right)\right)\right\rangle^{2}
$$

In the following subsections, we present the calculations of the Fano factor for the steady-state models illustrated in Fig. 1 of the Main Text. 


\section{A. Drift-diffusion in a triangular potential}

The system is defined on a one dimensional segment $[0,1]$ with periodic boundary conditions. The potential $U(x)$ is triangular, so that the total force is constant in the intervals $\left[0, x^{*}\right]$ and $\left[x^{*}, 1\right]$. Let us call these two regions $A$ and $B$, respectively, and the corresponding total forces $F_{A}=f-\mathrm{d} U_{A}(x) / \mathrm{d} x$ and $F_{B}=f-\mathrm{d} U_{B}(x) / \mathrm{d} x$. In order to calculate the long-time Fano factor Eq. (38) we need to compute the quantity

$$
\begin{aligned}
\sigma_{\tau}^{2} & =\frac{2}{k_{\mathrm{B}}^{2}} \int_{0}^{\infty} \mathrm{d} t^{\prime \prime}\left\langle v_{S}\left(X\left(t^{\prime}+t^{\prime \prime}\right)\right) v_{S}\left(X\left(t^{\prime}\right)\right)\right\rangle-\left\langle v_{S}\left(X\left(t^{\prime}\right)\right)\right\rangle^{2} \\
& =\frac{2 J^{4}}{D^{2}} \int_{0}^{\infty} \mathrm{d} t \int_{0}^{1} \mathrm{~d} x \int_{0}^{1} \mathrm{~d} y \frac{1}{P_{\mathrm{st}}^{2}(x)} \frac{1}{P_{\mathrm{st}}^{2}(y)}\left[P(x, t \mid y, 0) P_{\mathrm{st}}(y)-P_{\mathrm{st}}(x) P_{\mathrm{st}}(y)\right] \\
& =\frac{2 J^{4}}{D^{2}} \int_{0}^{\infty} \mathrm{d} t \int_{0}^{1} \mathrm{~d} x \int_{0}^{1} \mathrm{~d} y \frac{1}{P_{\mathrm{st}}^{2}(x)} \frac{1}{P_{\mathrm{st}}(y)}\left[P(x, t \mid y, 0)-P_{\mathrm{st}}(x)\right]
\end{aligned}
$$

where $P_{\text {st }}(x)$ is the steady-state probability density. The Fokker-Planck equations read

$$
\begin{array}{ll}
\partial_{t} P(x, t)=-\mu F_{A} \partial_{x} P+D \partial_{x}^{2} P & x \in\left[0, x^{*}\right] \\
\partial_{t} P(x, t)=-\mu F_{B} \partial_{x} P+D \partial_{x}^{2} P & x \in\left[x^{*}, 1\right] .
\end{array}
$$

Let us first compute $P_{\text {st }}(x)$. Solving Eq. (40) at steady state yields

$$
\begin{array}{ll}
P_{\mathrm{st}}(x)=\alpha_{1}+\alpha_{2} e^{F_{A} x /\left(k_{\mathrm{B}} T\right)} & x \in\left[0, x^{*}\right] \\
P_{\mathrm{st}}(x)=\alpha_{3}+\alpha_{4} e^{F_{B} x /\left(k_{\mathrm{B}} T\right)} & x \in\left[x^{*}, 1\right] .
\end{array}
$$

The four integration constants are determined by imposing 1) normalization of $P_{\mathrm{st}}(x)$, 2) conservation of current $\left.J_{A}=J_{B}, 3\right)$ continuity of probability in $x=x^{*}$, and 4$)$ periodic boundary condition $P_{\text {st }}(0)=P_{\text {st }}(1)$. Notice that all these conditions are linear in the integration constants. The explicit solution is

$$
\begin{aligned}
\alpha_{1} & =\frac{F_{A} F_{B}^{2}\left[e^{F_{B} x^{*} /\left(k_{\mathrm{B}} T\right)}-e^{\left(F_{B}+F_{A} x^{*}\right) /\left(k_{\mathrm{B}} T\right)}\right]}{\mathcal{N}} \\
\alpha_{2}= & \frac{F_{A} F_{B}\left(F_{B}-F_{A}\right)\left[e^{F_{B} /\left(k_{\mathrm{B}} T\right)}-e^{F_{B} x^{*} /\left(k_{\mathrm{B}} T\right)}\right]}{\mathcal{N}} \\
\alpha_{3}= & \frac{F_{A}^{2} F_{B}\left[e^{F_{B} x^{*} /\left(k_{\mathrm{B}} T\right)}-e^{\left(F_{B}+F_{A} x^{*}\right) /\left(k_{\mathrm{B}} T\right)}\right]}{\mathcal{N}} \\
\alpha_{4}= & \frac{F_{A} F_{B}\left(F_{A}-F_{B}\right)\left(e^{F_{A} x^{*} /\left(k_{\mathrm{B}} T\right)}-1\right)}{\mathcal{N}},
\end{aligned}
$$

with the normalization constant $\mathcal{N}=k_{\mathrm{B}} T\left(e^{F_{A} x^{*} /\left(k_{\mathrm{B}} T\right)}-1\right)\left(e^{F_{B} /\left(k_{\mathrm{B}} T\right)}-e^{F_{B} x^{*} /\left(k_{\mathrm{B}} T\right)}\right)\left(F_{A}-F_{B}\right)^{2}+$ $F_{A} F_{B}\left[e^{F_{B} x^{*} /\left(k_{\mathrm{B}} T\right)}-e^{\left(F_{B}+F_{A} x^{*}\right) /\left(k_{\mathrm{B}} T\right)}\right]\left(F_{A}-F_{A} x^{*}+F_{B} x^{*}\right)$.

Note that

$$
v_{S}(X(t))=\frac{k_{\mathrm{B}} J^{2}}{D P_{\mathrm{st}}^{2}(X(t))},
$$

with the current given by $J=\mu F_{A} \alpha_{1}=\mu F_{B} \alpha_{3}$, and $P_{\text {st }}(x)$ given by Eqs. (41) and (42). Its average equals

$$
\left\langle v_{S}\right\rangle=\frac{k_{\mathrm{B}} J^{2}}{D}\left\langle\frac{1}{P_{\mathrm{st}}^{2}}\right\rangle=\frac{k_{\mathrm{B}} J^{2} \gamma}{D}
$$

where we have defined the quantity

$$
\begin{aligned}
\gamma & =\int_{0}^{1} \mathrm{~d} x \frac{1}{P_{\mathrm{st}}(x)} \\
& =\frac{x^{*}}{\alpha_{1}}+k_{\mathrm{B}} T \frac{\ln \left(\alpha_{1}+\alpha_{2}\right)-\ln \left(\alpha_{1}+\alpha_{2} e^{F_{A} x^{*} / D}\right)}{\alpha_{1} F_{A}} \\
& +\frac{1-x^{*}}{\alpha_{3}}+k_{\mathrm{B}} T \frac{\ln \left(\alpha_{3}+\alpha_{4} e^{F_{B} x^{*} / D}\right)-\ln \left(\alpha_{3}+\alpha_{4} e^{F_{B} / D}\right)}{\alpha_{3} F_{B}}
\end{aligned}
$$


Let us now define

$$
f(x, t)=\int_{0}^{1} \mathrm{~d} y \frac{P(x, t \mid y, 0)-P_{\mathrm{st}}(x, t)}{P_{\mathrm{st}}(y)} .
$$

The function $f(x, t)$ is a solution of the Fokker-Planck Eq. (40) with initial condition $f(x, 0)=1 / P_{\text {st }}(x)-\gamma P_{\text {st }}(x)$. Notice that $f(x, t)$ is not a probability distribution as $\int \mathrm{d} x f(x, t)=0 \forall t$. We also introduce an additional function $\phi(x)=\int_{0}^{\infty} \mathrm{d} t f(x, t)$. Integrating the Fokker-Planck equation in time, we find that $\phi(x)$ obeys

$$
\begin{aligned}
-\mu F_{A} \partial_{x} \phi+D \partial_{x}^{2} \phi & =-f(x, 0)=-1 / P_{\mathrm{st}}(x)+\gamma P_{\mathrm{st}}(x) & & x \in\left[0, x^{*}\right] \\
-\mu F_{B} \partial_{x} \phi+D \partial_{x}^{2} \phi & =-f(x, 0)=-1 / P_{\mathrm{st}}(x)+\gamma P_{\mathrm{st}}(x) & & x \in\left[x^{*}, 1\right] .
\end{aligned} .
$$

The solution to this equation is

$$
\begin{aligned}
\phi(x) & =\beta_{1}+\frac{1}{\alpha_{1}^{2} \mu F_{A}^{2}}\left\{e^{F_{A} x /\left(k_{\mathrm{B}} T\right)}\left[\alpha_{1}^{2} \beta_{2} \mu k_{\mathrm{B}} T F_{A}+\alpha_{1}^{2} \alpha_{2} \gamma\left(F_{A} x-k_{\mathrm{B}} T\right)-\alpha_{2} k_{\mathrm{B}} T \ln \left(\alpha_{2}+\alpha_{1} e^{-F_{A} x /\left(k_{\mathrm{B}} T\right)}\right)\right]\right. \\
& \left.-\alpha_{1}\left(\gamma \alpha_{1}^{2}-1\right) F_{A} x-k_{\mathrm{B}} T \alpha_{1} \ln \left(\alpha_{1}+\alpha_{2} e^{F_{A} x /\left(k_{\mathrm{B}} T\right)}\right)\right\} \quad x \in\left[0, x^{*}\right] \\
\phi(x) & =\beta_{3}+\frac{1}{\alpha_{3}^{2} \mu F_{B}^{2}}\left\{e^{F_{B} x / k_{\mathrm{B}} T}\left[\alpha_{3}^{2} \beta_{4} \mu k_{\mathrm{B}} T F_{B}+\alpha_{3}^{2} \alpha_{4} \gamma\left(F_{B} x-k_{\mathrm{B}} T\right)-\alpha_{4} k_{\mathrm{B}} T \ln \left(\alpha_{4}+\alpha_{3} e^{-F_{B} x /\left(k_{\mathrm{B}} T\right)}\right)\right]\right. \\
& \left.-\alpha_{3}\left(\gamma \alpha_{3}^{2}-1\right) F_{B} x-k_{\mathrm{B}} T \alpha_{3} \ln \left(\alpha_{3}+\alpha_{4} e^{F_{B} x /\left(k_{\mathrm{B}} T\right)}\right)\right\} \quad x \in\left[x^{*}, 1\right] .
\end{aligned}
$$

The four integration constants $\beta_{i}, i=1 \ldots 4$ can be determined using similar conditions we imposed for the stationary distribution (41).

Defining the quantity $\psi=\int_{0}^{1} \mathrm{~d} x \phi(x) / P_{\mathrm{st}}^{2}(x)$, Eq. (39) can be rewritten as

$$
\frac{\sigma_{\tau}^{2}}{\langle\tau\rangle}=\frac{2 J^{2} \psi}{D k_{\mathrm{B}} \gamma}
$$

The integral in the definition of $\psi$ can be computed analytically, but yields a lengthy expression involving special functions that is hard to evaluate numerically. For this reason, the theoretical line in Fig. 4 of the Main Text was obtained from Eq. (49) by numerically integrating the expression for $\psi$.

\section{B. 2D transport in a force field}

We consider the following two-dimensional dynamics coordinate

$$
\begin{aligned}
\frac{\mathrm{d} X}{\mathrm{~d} t} & =\mu F(Y)+\sqrt{2 D} \xi_{X} \\
\frac{\mathrm{d} Y}{\mathrm{~d} t} & =\sqrt{2 D} \xi_{Y},
\end{aligned}
$$

with the non-conservative force $F(y)=f \cos (2 \pi y)$. This case is considerably simpler than that of the previous section as the stationary distributon is homogeneous, so that

$$
v_{S}(X(t), Y(t))=v_{S}(Y(t))=\frac{k_{\mathrm{B}} \mu^{2} F^{2}(Y(t))}{D}
$$

and its average its given by

$$
\left\langle v_{S}\right\rangle=k_{\mathrm{B}} \int_{0}^{1} \frac{\mu^{2} F^{2}(y)}{D} \mathrm{~d} y=\frac{\mu f^{2}}{2 T} .
$$

Moreover, since the force depends only on the $y$ position, the correlation entering the Green-Kubo formula can be calculated and gives

$$
\begin{aligned}
\left\langle v_{S}(y(0)) v_{S}(y(t))\right\rangle & =\frac{k_{\mathrm{B}}^{2} \mu^{4}}{D^{2}} \int_{0}^{1} d y \int_{-\infty}^{\infty} d \Delta y F^{2}(y) F^{2}(y+\Delta y) \frac{e^{-\frac{\Delta y^{2}}{4 D t}}}{\sqrt{4 \pi D t}} \\
& =\frac{\mu^{2} f^{4}}{4 T^{2}}\left[1+\frac{1}{2} e^{-16 \pi^{2} D t}\right] .
\end{aligned}
$$

Substituting this expression in Eq. (38) we obtain

$$
\frac{\sigma_{\tau}^{2}}{\langle\tau\rangle}=\frac{f^{2}}{32 \pi^{2} k_{\mathrm{B}}^{2} T^{2}}
$$




\section{Chiral active Brownian motion}

This model is defined by the set of equations

$$
\begin{aligned}
\mathrm{d} X / \mathrm{d} t & =\mu f \cos (\phi)+\sqrt{2 D} \xi_{x} \\
\mathrm{~d} Y / \mathrm{d} t & =\mu f \sin (\phi)+\sqrt{2 D} \xi_{y} \\
\mathrm{~d} \phi / \mathrm{d} t & =\mu_{\phi} \omega+\sqrt{2 D_{\omega}} \xi_{\omega} .
\end{aligned}
$$

In this case, the entropy drift is constant and given by

$$
v_{S}=\frac{k_{\mathrm{B}} \mu^{2} f^{2}}{D}+\frac{k_{\mathrm{B}} \mu_{\phi}^{2} \omega^{2}}{D_{\phi}} .
$$

Therefore $\tau$ grows in a deterministic way, $\sigma_{\tau}^{2}=0$, the distribution of $S_{\text {tot }}$ is Gaussian and the Fano factor of $S_{\text {tot }}$ is equal to 2 .

\section{S4. FANO FACTOR OF ENTROPY PRODUCTION OUT OF STEADY STATE}

In this section, we derive the Fano factor equality out of steady state, given by Eq. (17) in the main text. We find the average of the squared entropy production from the expression for the stochastic entropy production Eq. (24), namely,

$$
\begin{aligned}
\frac{\left\langle S_{\mathrm{tot}}^{2}(t)\right\rangle}{k_{\mathrm{B}}^{2}} & =\left\langle\left[\int_{0}^{t} \mathrm{~d} t^{\prime}\left\{\frac{v_{S}\left(\vec{X}\left(t^{\prime}\right), t^{\prime}\right)}{k_{\mathrm{B}}}-2 \partial_{t^{\prime}} \ln P\left(\vec{X}\left(t^{\prime}\right), t^{\prime}\right)+\xi_{S}\left(t^{\prime}\right) \sqrt{\frac{2 v_{S}\left(\vec{X}\left(t^{\prime}\right), t^{\prime}\right)}{k_{\mathrm{B}}}}\right\}\right]^{2}\right\rangle \\
& =\left\langle\tau^{2}\right\rangle+2\langle\tau\rangle+4\left\langle\left[\int_{0}^{t} \mathrm{~d} t^{\prime} \partial_{t^{\prime}} \ln P\left(\vec{X}\left(t^{\prime}\right), t^{\prime}\right)\right]^{2}\right\rangle-4\left\langle\tau(t) \int_{0}^{t} \mathrm{~d} t^{\prime} \partial_{t^{\prime}} \ln P\left(\vec{X}\left(t^{\prime}\right), t^{\prime}\right)\right\rangle \\
& +2 \sqrt{2}\left\langle\int_{0}^{t} \mathrm{~d} t^{\prime} \int_{0}^{t} \mathrm{~d} t^{\prime \prime} \xi_{S}\left(t^{\prime \prime}\right) \sqrt{\frac{v_{S}\left(\vec{X}\left(t^{\prime \prime}\right), t^{\prime \prime}\right)}{k_{\mathrm{B}}}}\left[\frac{v_{S}\left(\vec{X}\left(t^{\prime}\right), t^{\prime}\right)}{k_{\mathrm{B}}}-2 \partial_{t^{\prime}} \ln P\left(\vec{X}\left(t^{\prime}\right), t^{\prime}\right)\right]\right\rangle .
\end{aligned}
$$

We first evaluate the term in the last line using Doob's h-transform, as in Eqs. (30)-(36). Following this procedure, we obtain

$$
\begin{aligned}
& 2 \sqrt{2} \int_{0}^{t} \mathrm{~d} t^{\prime} \int_{0}^{t} \mathrm{~d} t^{\prime \prime}\left\langle\xi_{S}\left(t^{\prime \prime}\right) \sqrt{v_{S}\left(\vec{X}\left(t^{\prime \prime}\right), t^{\prime \prime}\right)}\left[\frac{v_{S}\left(\vec{X}\left(t^{\prime}\right), t^{\prime}\right)}{k_{\mathrm{B}}^{3 / 2}}-\frac{2}{\sqrt{k_{\mathrm{B}}}} \partial_{t^{\prime}} \ln P\left(\vec{X}\left(t^{\prime}\right), t^{\prime}\right)\right]\right\rangle \\
& =4 \int_{0}^{t} \mathrm{~d} t^{\prime} \int_{0}^{t^{\prime}} \mathrm{d} t^{\prime \prime}\left\langle\partial_{t^{\prime \prime}} \ln P\left(\vec{X}\left(t^{\prime \prime}\right), t^{\prime \prime}\right)\left[\frac{v_{S}\left(\vec{X}\left(t^{\prime}\right), t^{\prime}\right)}{k_{\mathrm{B}}}-2 \partial_{t^{\prime}} \ln P\left(\vec{X}\left(t^{\prime}\right), t^{\prime}\right)\right]\right\rangle
\end{aligned}
$$

Substituting this expression into Eq. (57) yields

$$
\frac{\left\langle S_{\mathrm{tot}}^{2}(t)\right\rangle}{k_{\mathrm{B}}^{2}}=\left\langle\tau^{2}(t)\right\rangle+2\langle\tau(t)\rangle-\frac{4}{k_{\mathrm{B}}} \int_{0}^{t} \mathrm{~d} t^{\prime} \int_{0}^{t^{\prime}} \mathrm{d} t^{\prime \prime}\left\langle\partial_{t^{\prime \prime}} \ln P\left(\vec{X}\left(t^{\prime \prime}\right), t^{\prime \prime}\right) v_{S}\left(\vec{X}\left(t^{\prime}\right), t^{\prime}\right)\right\rangle
$$

which leads to Eqs. (17), (18), and (19) in the Main Text.

\section{S5. FANO FACTOR OF ENTROPY PRODUCTION FOR A QUENCH OF AN HARMONIC TRAP}

In this section, we briefly present details of the numerical simulations shown in Fig. 5 of the Main Text. We consider a system described by the one dimensional Fokker-Planck equation

$$
\partial_{t} P(x, t)=\partial_{x}\left[\mu \kappa_{f} x P(x, t)+D \partial_{x} P(x, t)\right]
$$

The system is initially prepared at thermal equilibrium with a stiffness $\kappa_{i}$ and then instantaneously quenched to the stiffness $\kappa_{f}$. The Fano factor of total entropy production at long times, shown in Fig. 5 in the Main Text, is computed from simulations by the usual definition of total entropy production $S_{\text {tot }}=(W-\Delta F) / T$ so that

$$
\frac{\sigma_{S_{\text {tot }}}^{2}}{k_{\mathrm{B}}\left\langle S_{\text {tot }}\right\rangle}=\frac{\sigma_{W}^{2}}{k_{\mathrm{B}} T(\langle W\rangle-\Delta F)}
$$


In this case, the work is simply given by the instantaneous change in energy due to the quench

$$
W=\left(\frac{\partial U}{\partial k}\right) \Delta k=\frac{1}{2}\left(\kappa_{f}-\kappa_{i}\right) x^{2}(t=0)
$$

Averaging over the initial condition, the mean work is

$$
\langle W\rangle=\int_{-\infty}^{\infty} \mathrm{d} x P(x, 0) \frac{1}{2}\left(\kappa_{f}-\kappa_{i}\right) x^{2}=\frac{k_{\mathrm{B}} T}{2} \frac{\left(\kappa_{f}-\kappa_{i}\right)}{\kappa_{i}},
$$

where we used the fact that $P(x, 0)$ is Gaussian with mean 0 and variance $k_{\mathrm{B}} T / \kappa_{i}$. Similarly we have

$$
\left\langle W^{2}\right\rangle=\int_{-\infty}^{\infty} \mathrm{d} x P(x, 0)\left(\frac{1}{2}\left(\kappa_{f}-\kappa_{i}\right) x^{2}\right)^{2}=\frac{3\left(k_{\mathrm{B}} T\right)^{2}}{4} \frac{\left(\kappa_{f}-\kappa_{i}\right)^{2}}{\kappa_{i}^{2}},
$$

so that

$$
\sigma_{W}^{2}=\left\langle W^{2}\right\rangle-\langle W\rangle^{2}=\frac{\left(k_{\mathrm{B}} T\right)^{2}}{2} \frac{\left(\kappa_{f}-\kappa_{i}\right)^{2}}{\kappa_{i}^{2}} .
$$

Finally, for this protocol we simply have

$$
\Delta F=\frac{k_{\mathrm{B}} T}{2} \log \left(\kappa_{f} / \kappa_{i}\right)
$$

Plugging these expressions into Eq. (67) we obtain an exact expression for the Fano factor:

$$
\frac{\sigma_{S_{\text {tot }}}^{2}}{k_{\mathrm{B}}\left\langle S_{\text {tot }}\right\rangle}=\frac{\left(\kappa_{f} / \kappa_{i}-1\right)^{2}}{\left(\kappa_{f} / \kappa_{i}-1\right)-\log \left(\kappa_{f} / \kappa_{i}\right)} .
$$

To compare Eq. (67) with Eq. (17) in the Main Text we recall that Eq. (60) describes an Ornstein-Uhlenbeck process, for which the propagator reads

$$
P\left(x, t \mid x^{\prime}, t^{\prime}\right)=\sqrt{\frac{\kappa_{f}}{2 \pi k_{\mathrm{B}} T\left(1-e^{-2 \mu \kappa_{f}\left(t-t^{\prime}\right)}\right)}} \exp \left[-\frac{\kappa_{f}\left(x-e^{-\mu \kappa_{f}\left(t-t^{\prime}\right)} x^{\prime}\right)^{2}}{2 k_{\mathrm{B}} T\left(1-e^{-2 \mu \kappa_{f}\left(t-t^{\prime}\right)}\right)}\right] .
$$

Notice that the propagator is Gaussian. The system is initially in equilibrium with a different stiffness $\kappa_{i}$, so that its initial distribution is Gaussian with mean zero and variance $\sigma_{i}^{2}=k_{\mathrm{B}} T / \kappa_{i}$. Integrating Eq. (68) over such initial condition, we find that the distribution of the system remains Gaussian at all times:

$$
P(x, t)=\frac{1}{\sqrt{2 \pi \sigma^{2}(t)}} \exp \left[-x^{2} / 2 \sigma^{2}(t)\right]
$$

where

$$
\sigma^{2}(t)=\sigma_{f}^{2}+\left(\sigma_{i}^{2}-\sigma_{f}^{2}\right) e^{-2 \mu \kappa_{f} t},
$$

and $\sigma_{f}^{2}=k_{\mathrm{B}} T / \kappa_{f}$. We can now write the probability current as

$$
J(x, t)=-\mu \kappa_{f} x P(x, t)-D \partial_{x} P(x, t) \quad .
$$

The corresponding entropic drift is

$$
\begin{aligned}
\frac{v_{S}(X(t), t)}{k_{\mathrm{B}}} & =\frac{J^{2}(X(t), t)}{D P^{2}(X(t), t)} \\
& =\left(\frac{\kappa_{f}}{\kappa_{i}}-1\right)^{2} e^{-4 \mu \kappa_{f} t} \frac{D X^{2}(t)}{\sigma^{4}(t)} .
\end{aligned}
$$

Similarly, one can show that

$$
\partial_{t} \ln P(x, t)=\mu \kappa_{f}\left[x^{2}-\sigma^{2}(t)\right] \frac{\left(\sigma_{f}^{2}-\sigma_{i}^{2}\right) e^{-2 \mu \kappa_{f} t}}{\sigma^{4}(t)} .
$$

Using Eqs. (72) and (73), one can estimate the quantities $\sigma_{\tau}^{2}$ and $\Omega$ by calculating suitable averages in numerical simulations of the process. The curves in Figs. $5 \mathrm{a}$ and $5 \mathrm{~b}$ in the Main Text have been computed by this method. 